\title{
Tanshinone II is a potent candidate for treatment of lipopolysaccharide-induced acute lung injury in rat model
}

\author{
LING LI, YONG-GANG ZHANG, YU-FEI TAN, JING-JING ZHAO, HUA-RU ZHANG and BING ZHAO \\ Department of Respiratory and Critical Care, Zhumadian Central Hospital, Zhumadian, Henan 463000, P.R. China
}

Received August 18, 2016; Accepted September 7, 2017

DOI: 10.3892/ol.2017.7581

\begin{abstract}
The present study aimed to investigate the effect of tanshinone II, isolated from Salvia miltiorrhiza Bunge, on lipopolysaccharide (LPS)-induced acute lung injury (ALI) in rats. Male Sprague-Dawley rats were divided into three groups: Control, LPS and tanshinone II. Animals in the tanshinone II and LPS groups were administered $10 \mathrm{mg} / \mathrm{kg}$ LPS, whereas those in the control group received an equal volume of normal saline. Tanshinone II treatment group were injected with $30 \mathrm{~nm} / \mathrm{kg}$ tanshinone II at $1 \mathrm{~h}$ after LPS administration. The results revealed that LPS administration increased the bronchoalveolar lavage fluid protein concentration significantly compared with the control group. However, tanshinone II treatment significantly inhibited the LPS-induced increase in protein level. Treatment of the LPS-administered rats with tanshinone II prevented the formation of pulmonary edema, which was evidenced by low values for wet to dry lung weight ratio. The activity of myeloperoxidase and expression of malondialdehyde were significantly lower in lung homogenates from the tanshinone II group compared with the LPS group. Furthermore, tanshinone II treatment inhibited the expression of tumor necrosis factor- $\alpha$ and interleukin- 6 in the blood plasma. Tissue sections of the tanshinone II group exhibited normal morphology and absence of neutrophil accumulation. However, in the LPS group, neutrophils accumulated and penetrated into the pulmonary tissues. These results suggested that tanshinone II protects the rats from LPS-induced ALI. Therefore tanshinone II may have clinical applications in the treatment of ALI.
\end{abstract}

\section{Introduction}

Acute lung injury (ALI) is a serious health disorder that leads to dysfunction of the respiratory system and has a mortality rate

Correspondence to: Dr Bing Zhao, Department of Respiratory and Critical Care, Zhumadian Central Hospital, 747 Zhonghua Road, Zhumadian, Henan 463000, P.R. China

E-mail: zhaobing2134@gmail.com

Key words: lung injury, edema, neutrophils, alveolar space, prevention of $>50 \%$ (1). The disease is a clinical challenge for researchers worldwide due to the high rate of mortality. The factors responsible for inducing ALI include inhalation of the endotoxins from gram-negative bacteria and sepsis. Administration of lipopolysaccharide (LPS) has been used to prepare ALI animal models for use in numerous studies (2-6). It has been reported that LPS treatment in rats induces inflammatory reactions, leading to the development of injury and ultimately lung dysfunction (7). ALI in animals is associated with degeneration of lung tissues, enhancement in the protein concentration and fluid, developing into edema and breakage of capillary walls (8). LPS administration in rats causes accumulation and penetration of neutrophils into pulmonary tissues, which then secrete oxidants leading to lung injury $(9,10)$. These findings are supported by the fact that treatment of LPS-administered animals with oxidant scavengers can protect against lung injury (11).

Natural products have shown potential in the treatment of numerous diseases and therefore many of these are used either directly as drugs or modified to develop as chemotherapeutic agents (12). Danshen (Salvia miltiorrhiza Bunge) is used in traditional Chinese medicine for the treatment of diseases related to blood flow $(13,14)$. Phytochemical analysis of Danshen plant has led to the isolation of a large number of compounds. The major compounds are phenolics and diterpenoids, including tanshinone II (15). It is reported that tanshinone II is the most biologically active compound isolated from Salvia miltiorrhiza (16). Tanshinone II treatment has been reported to cause apoptosis in various types of carcinoma cell lines $(16,17)$. The present study was performed to investigate the effect of tanshinone II on LPS-induced ALI in rats. The results demonstrated that tanshinone II exhibits protective effects against LPS-induced ALI in rats.

\section{Materials and methods}

Chemicals and reagents. Tanshinone II (purity 98\%), dimethyl sulphoxide (DMSO), LPS and other common chemicals were obtained from Sigma-Aldrich (Merck KGaA, Darmstadt, Germany). Stock solution of tanshinone II was prepared in DMSO and stored until use in experiments at $10^{\circ} \mathrm{C}$.

Animals. Thirty male Sprague-Dawley rats (8-week-old) weighing 200-220 g were obtained from the Laboratory Animal Center, Academy of Military Medical Sciences 
(Beijing, China). The animals were housed one week before the start of experiment under standard laboratory conditions at a stable temperature $\left(22-24^{\circ} \mathrm{C}\right)$, humidity $(55+5 \%)$ and a $12 / 12-\mathrm{h}$ light/dark cycle with food and water ad libitum. Animals were maintained as per the guidelines for Care and Use of Laboratory Animals of the National Institutes of Health and Academy of Military Medical Sciences (18). The study was approved by the Ethics Committee of Animal Experiments of the Zhumadian Central Hospital, Zhumadian, Henan China. Sodium pentobarbital (Bi Biotech India Pvt Ltd, New Delhi, India) was used to sacrifice the animals for extraction of lungs.

Treatment strategy. The animals were divided randomly into three groups of 10 animals each: Control, LPS and tanshinone II groups. Animals in the LPS and tanshinone II groups were administered $10 \mathrm{mg} / \mathrm{kg}$ body weight LPS intraperitoneally. Tanshinone II at a concentration of $30 \mu \mathrm{g} / \mathrm{kg}$ was injected intraperitoneally to the animals in the tanshinone II group at $1 \mathrm{~h}$ after administration of LPS. Animals in the normal control group received an equal volume of normal saline alone. On day 8 after administration of tanshinone II, the animals were sacrificed to extract the lungs and collect abdominal aorta blood samples. The weight of the lungs was recorded to calculate the lung wet/dry (W/D) ratio in the animals.

Lung W/D ratio. The lung W/D ratio was determined using a previously reported protocol (11). Briefly, the lungs were extracted, followed by separation of the right lung. The right lung was weighed immediately after extraction to determine its wet weight followed by drying for 5 min under microwaves at low power $(200 \mathrm{~W})$ to measure the dry weight. The W/D ratio was calculated as follows: $\mathrm{W} / \mathrm{D}=$ weight wet/weight dry.

Bronchoalveolar lavage. Left lung of the animals was washed with normal saline three times (5 min each time) and the washing fluid was collected for determination of protein concentration. An ELISA protein kit (IL-6, SEA079Hu; and TNF- $\alpha$, E90133Hu; Uscn Life Sciences, Inc., Wuhan, China) was used to determine the concentration of proteins according to manufacturer's protocol.

Analysis of myeloperoxidase (MPO) and malondialdehyde (MDA) activities in lung homogenates. Lung tissues were lysed using RIPA buffer (Cell Signaling Technology, Inc., Danvers, MA, USA) and a DC protein assay (Bio-Rad Laboratories, Inc., Hercules, CA, USA) was used for the determination of protein concentration in the homogenates. The cellular lysates were centrifuged at $15,000 \mathrm{rpm}$ for $15 \mathrm{~min}$ at $4^{\circ} \mathrm{C}$, and the supernatants were harvested and mixed with loading buffer. MPO and MDA activity was determined using MPO ELISA kit (cat. no. DRE 30329; Xinfan Biomart Co., Ltd., Shanghai, China) and MDA kit (cat. no. ab118970; Abcam; Cambridge, $\mathrm{UK}$ ) respectively, according to the manufacturer's protocols.

Tumor necrosis factor (TNF)- $\alpha$ and interleukin (IL)-6 levels in blood samples. Blood samples collected from the aortic artery in the abdominal region of the rats were anti-coagulated using EDTA. The blood was then centrifuged for $15 \mathrm{~min}$ at $4,000 \mathrm{xg}$ at $0^{\circ} \mathrm{C}$ to collect the plasma. The levels of TNF- $\alpha$ (cat. no. RAB0476) and IL-6 (cat. no. RAB0311; Sigma-Aldrich;
Merck $\mathrm{KGaA}$ ) in the plasma were determined using ELISA kits.

RNA interference. The lung tissue samples were transfected with siRNA sequences, as described previously (19). The siRNA sequences, 5'-ACUUUGCUGUAACCCUGUA (dTdT)-3' (sense) and 5'-UACAGGGUUACAGCAAAGU (dTdT)-3' (antisense) were used to silence Sirt1 expression.

Western blot analysis. Lung tissues were lysed by treatment with RIPA buffer (Cell Signaling Technology, Inc.) for $15 \mathrm{~min}$ at $0^{\circ} \mathrm{C}$ and lysates were centrifuged at $14,000 \mathrm{x}$ g for $20 \mathrm{~min}$ at $4^{\circ} \mathrm{C}$. The concentration of proteins in tissue lysates was detected using the $\mathrm{DC}^{\mathrm{TM}}$ protein assay (Bio-Rad, Laboratories, Inc., Hercules, CA, USA). The protein samples (10 $\mu \mathrm{g})$ were separated by SDS-PAGE using $7.5 \%$ polyacrylamide gels and transferred onto PVDF membranes (Millipore Corp., Bedford, MA, USA). The membranes were blocked for $1 \mathrm{~h}$ at room temperature in 5\% non-fat dry milk in TBS. The membranes were then subjected to immunodetection with a 1:1,000 dilution of respective primary antibodies against MMP9 (cat. no. 3852), iNOS (cat. no. 2982), IL-1 (cat. no. 5204), IL-6 (cat. no. 5216) and Sirt1 (cat. no. 2310; dilution 1:500, Cell Signaling Technology, Inc.) overnight at $4^{\circ} \mathrm{C}$. Following washing, the membranes were incubated with a 1:20,000 dilution of horseradish peroxidase (HRP)-conjugated goat anti-rabbit or anti-mouse secondary antibody (cat. no. 27647; Cell Signaling Technology, Inc.) for $1 \mathrm{~h}$ at room temperature. Antigen-antibody complexes were visualized with chemiluminescence HRP substrate (EMD Millipore, Billerica, MA, USA) and LAS-1000 image analyzer (Fuji Film, Tokyo, Japan).

Histochemical examination. A portion of the right lung was exposed to buffered formaldehyde $(3 \%)$ for $1 \mathrm{~h}$ at room temperature and then subjected to paraffin embedding. The paraffin-embedded lung tissues were cut into $2-\mu \mathrm{m}$ sections, followed by staining with hematoxylin and eosin at room temperature for $4 \mathrm{~h}$. The paraffin-embedded lung sections were deparaffinized by boiling in xylene followed by rehydration through a graded ethanol series (Japan Alcohol Trading Co., Ltd., Tokyo, Japan). The sections were treated for $15 \mathrm{~min}$ with $3 \%$ hydrogen peroxide solution for quenching the activity of endogenous peroxidase. The sections were examined under a light microscope and assessed for alterations in lung morphology.

Statistical analysis. Data are expressed as the mean \pm standard deviation. Comparisons among the groups was performed using one-way analysis of variance with the Bonferroni post-hoc test. Statistical analyses were performed using SPSS v17 software (SPSS, Inc., Chicago, IL, USA). P $<0.05$ was considered to indicate a statistically significant difference.

\section{Results}

Tanshinone II inhibits expression of proteins present in bronchoalveolar lavage fluid (BALF). Analysis of the BALF revealed a significant increase in the protein level in LPS-administered rats compared with the control group (Fig. 1; P<0.02). Tanshinone II treatment exhibited a significant 


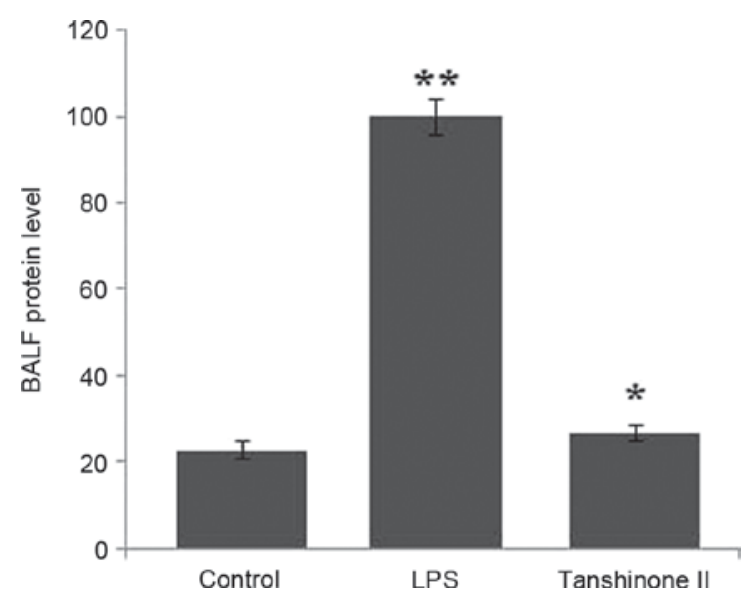

Figure 1. Effect of tanshinone II on LPS-induced BALF protein concentration in rats. The experiments were performed in triplicate. ${ }^{*} \mathrm{P}<0.05$ and ${ }^{* *} \mathrm{P}<0.02$ vs. control group. LPS, lipopolysaccharide; BALF, bronchoalveolar lavage fluid.

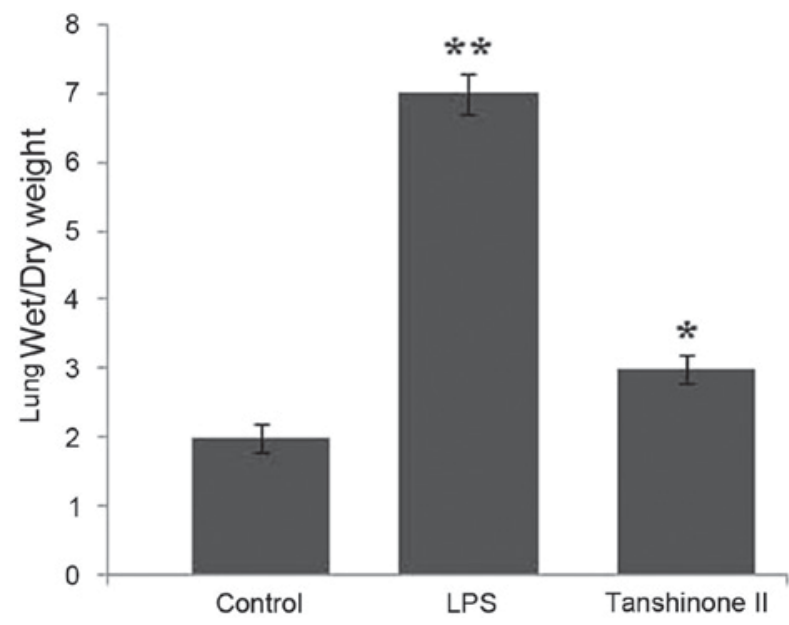

Figure 2. Effect of tanshinone II on the LPS-induced ratio of wet to dry lung weight in rats. ${ }^{*} \mathrm{P}<0.02$ and ${ }^{* *} \mathrm{P}<0.01$ vs. control. LPS, lipopolysaccharide.

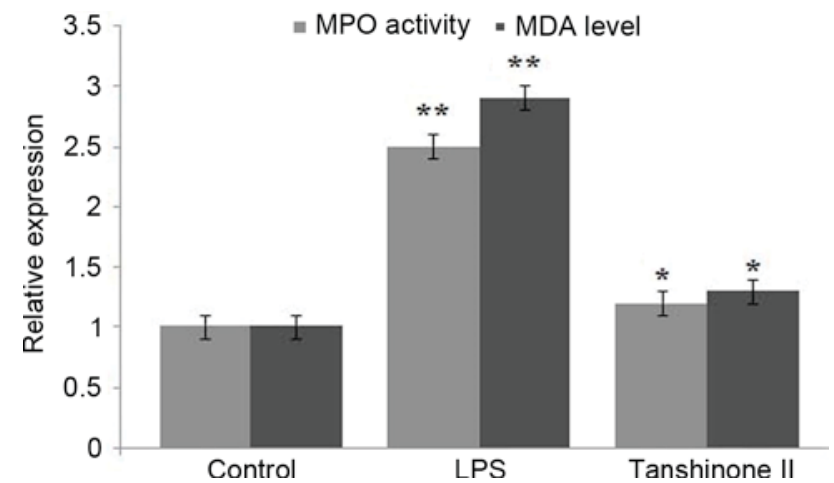

Figure 3. Effect of tanshinone II on MPO activity and MDA level in LPS-induced rats. ${ }^{*} \mathrm{P}<0.05$ and ${ }^{* *} \mathrm{P}<0.02$ vs. control. MPO, myeloperoxidase; MDA, malondialdehyde; LPS, lipopolysaccharide.

$(\mathrm{P}<0.05)$ inhibitory effect on the level of proteins in the BALF of LPS-administered rats. The concentration of proteins in LPS-administered rats was reduced to the level of the control group at $30 \mu \mathrm{g} / \mathrm{kg}$ concentration of tanshinone II (Fig. 1).

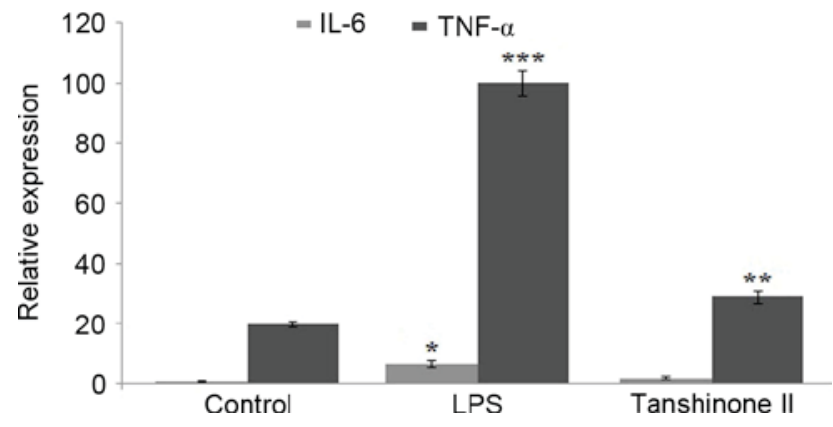

Figure 4. Effect of tanshinone II treatment on the expression levels of TNF- $\alpha$ and IL-6 in the blood samples of LPS-induced rats. ${ }^{*} \mathrm{P}<0.05,{ }^{* *} \mathrm{P}<0.02$ and ${ }^{* * * *} \mathrm{P}<0.02$ vs. control. TNF, tumor necrosis factor; IL, interleukin; LPS, lipopolysaccharide.

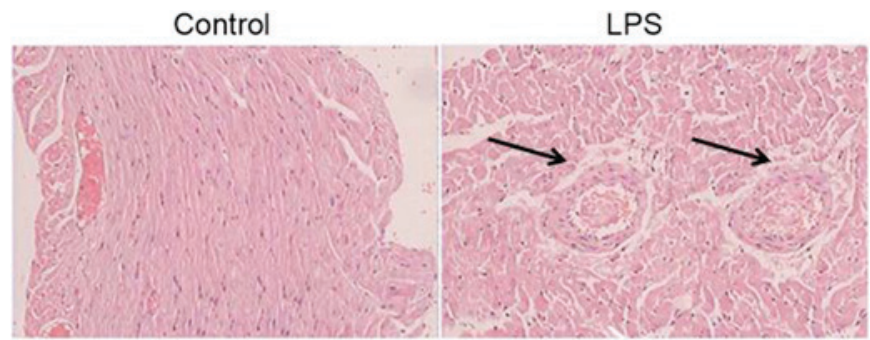

Tanshinone II

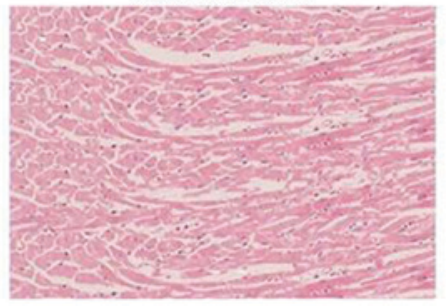

Figure 5. Alterations in the lung tissues of LPS-administered rats. Tissue sections were stained with hematoxylin and eosin and examined under a light microscope (magnification, x200). LPS, lipopolysaccharide. The black arrow indicates accumulation of neutrophils.

Effects of tanshinone II on lung W/Dratio. The lung W/D ratio in the control, LPS and tanshinone II groups were compared. The data revealed that LPS administration significantly increased the lung W/D ratio compared with the rats in the control group (P<0.05; Fig. 2). Treatment of the LPS-administered rats with $30 \mu \mathrm{g} / \mathrm{kg}$ concentration of tanshinone II significantly $(\mathrm{P}<0.02)$ inhibited the increase in lung W/D ratio (Fig. 2). The ratio of wet and dry weight in the rats of tanshinone II treatment and control groups was nearly similar.

Tanshinone II inhibited MPO activity and MDA level in $L P S$-administered rats. LPS administration significantly enhanced the level of MPO activity in rats compared with those in the control group (Fig. 3; $\mathrm{P}<0.03$ ). Tanshinone II treatment of the LPS-administered rats caused a significant $(\mathrm{P}<0.02)$ inhibition of LPS-induced increase in activity of MPO (Fig. 3). Analysis of MDA activity indicated a significantly higher level in LPS-administered rats compared with the control group (Fig. 3; P<0.03). However, treatment of the LPS-administered rats with $30 \mu \mathrm{g} / \mathrm{kg}$ concentration of tanshinone II caused a 


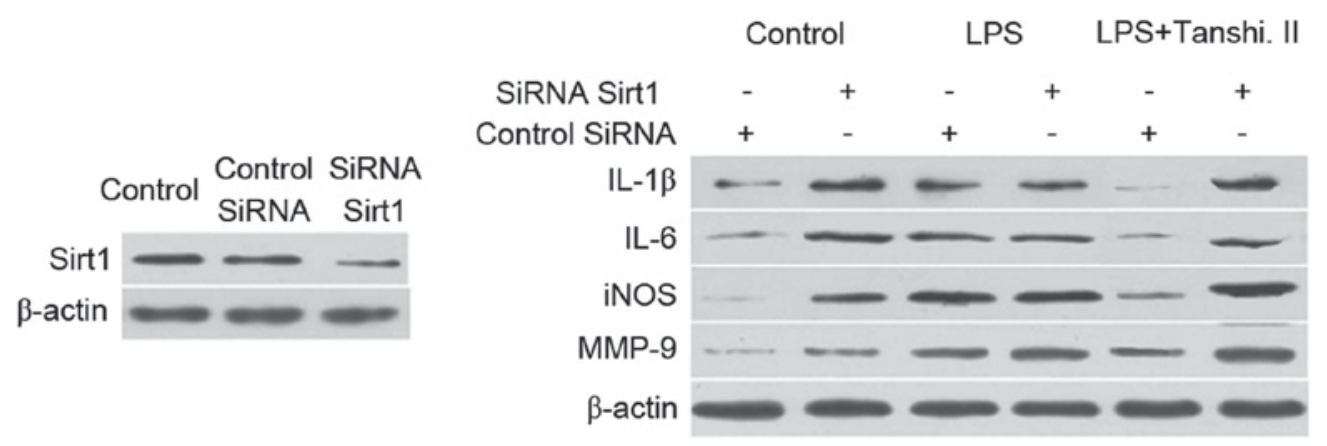

Figure 6. Tanshinone II prevents inflammation through targeting Sirt1 in LPS-induced rats. Lung tissues were transfected with siRNA targeting Sirt1. The expression of MMP-9, iNOS, IL-1 $\beta$ and IL-6 proteins in the lung tissues of rats was examined using western blot analysis. LPS, lipopolysaccharide; Tanshi. II, tanshinone II; siRNA, small interfering RNA; MMP, matrix metalloproteinase; iNOS, inducible nitric oxide synthase; IL, interleukin.

significant $(\mathrm{P}<0.05)$ decrease in MDA and reduced its level to that observed in the control group (Fig. 3).

Inhibition of TNF- $\alpha$ and IL-6 in blood samples of LPS-administered rats by tanshinone II. Blood samples collected from the rats were analyzed for the concentration of TNF- $\alpha$ and IL-6. The results revealed a significant increase in the level of TNF- $\alpha(\mathrm{P}<0.02)$ and IL-6 $(\mathrm{P}<0.05)$ on LPS administration compared with the control (Fig. 4). However, treatment of the LPS-administered rats with tanshinone II markedly reduced the level of TNF- $\alpha$ and IL- 6 compared with the LPS group (Fig. 4).

Tanshinone II exhibits a protective effect against LPS-induced pulmonary alterations. Light microscopy examination of the pulmonary tissues revealed accumulation of neutrophils and degradation of cells in LPS-administered animals (Fig. 5). Pulmonary tissues were intact without any appearance of degradation in the LPS-administered rats treated with tanshinone II. No evident symptoms of LPS administration were observed in tanshinone II treated rats (Fig. 5).

Tanshinone II prevents inflammation by targeting Sirt1 expression. Administration of small interfering RNA (siRNA) to inhibit the expression of Sirt1 in rats was performed. A marked reduction in the expression of gene corresponding to Sirt1 was observed after 48 h of siRNA administration (Fig. 6). Inhibition of matrix metalloproteinase (MMP)-9, inducible nitric oxide synthase (iNOS), IL-1 $\beta$ and IL- 6 expression was attenuated after tanshinone II treatment was attenuated on administration of rats with siRNA corresponding to Sirt1 (Fig. 6).

\section{Discussion}

ALI is a serious health problem detected frequently in developing countries and the rate of associated mortality is very high (20). The characteristic features of ALI are enhanced concentration of proteins, increased lung W/D ratio, and accumulation and penetration of neutrophils in pulmonary tissues (21). In the present study, the effect of tanshinone II on ALI in an LPS-induced lung injury rat model was analyzed. Administration of LPS induces cellular inflammatory processes and subsequent pulmonary injury in animals $(22,23)$. The present results demonstrated that LPS administration enhanced the concentration of proteins in pulmonary fluid of rats. Tanshinone II exhibited an inhibitory effect on the increase of pulmonary protein content in LPS-induced rats. Comparison of the lung W/D ratio in the LPS and control group indicated that LPS administration caused pulmonary edema in the rats. Treatment of the rats with tanshinone II inhibited LPS-induced lung edema, which was evidenced by similar lung W/D ratios in the tanshinone II and control groups. Neutrophils secrete an enzyme, MMP-9, which has been demonstrated to induce degradation of tissues by producing oxidative species (24). The present results revealed that tanshinone II treatment of LPS-induced rats reduced the expression of MMP-9 in the blood samples. These findings were further confirmed by histopathological examination of pulmonary tissues in tanshinone II-treated rats. Accumulation of neutrophils and tissue degradation was reduced in the LPS-induced rats treated with tanshinone II. The level of MDA in the blood samples of LPS-induced rats was also reduced by treatment with tanshinone II, indicating inhibition of peroxidation of membrane lipids (25).

TNF- $\alpha$ and ILs serve critical functions in proinflammatory activity and have been identified to increase the rate of mortality in animals (26). The present results demonstrated that tanshinone II treatment in the LPS-induced rats inhibited the expression of TNF- $\alpha$ and IL- 6 in blood samples. The level of these cytokines was significantly promoted in the rats on administration of LPS. Inflammatory processes or LPS administration induces expression of Sirt1 in cells $(27,28)$. In the present study, administration of siRNA against Sirt1 resulted in a significant reduction in the protein expression of Sirt1. Tanshinone II treatment inhibited the expression of MMP-9, iNOS, IL-1 $\beta$ and IL-6, and this effect was attenuated by administration of siRNA against Sirt1. These results suggest that tanshinone II inhibits LPS-induced lung injury through targeting Sirt1.

In summary, the present study demonstrates that tanshinone II inhibits LPS-induced lung injury in the rats by inhibiting TNF- $\alpha$ and IL-6 expression through targeting Sirt1. It also inhibits the LPS-induced increase in activity of MPO and level of MDA. Therefore, tanshinone II may be of therapeutic importance for the treatment of ALI. However, further studies need to be performed to understand the mechanism of action of tanshinone II clearly. 


\section{References}

1. Herridge MS, Cheung AM, Tansey CM, Matte-Martyn A, Diaz-Granados N, Al-Saidi F, Cooper AB, Guest CB, Mazer CD, Mehta S, et al: One-year outcomes in survivors of the acute respiratory distress syndrome. N Engl J Med 348: 683-693, 2003

2. Chung YJ, Jarvis B and Pestka J: Modulation of lipopolysaccharide-induced proinflammatory cytokine production by satratoxins and other macrocyclic trichothecenes in the murine macrophage. J Toxicol Environ Health A 66: 379-391, 2003.

3. Brandolini L, Asti C, Ruggieri V, Intilangelo A, Pellegrini L, ChiusaroliR,CaselliGFandBertiniR:Lipopolysaccharide-induced lung injury in mice. II. Evaluation of functional damage in isolated parenchyma strips. Pulm Pharmacol Ther 13: 71-78, 2000.

4. Bucher M and Taeger K: Endothelin-receptor gene-expression in rat endotoxemia. Intensive Care Med 28: 642-647, 2002.

5. Emery DA, Nagaraja KV, Sivanandan V, Lee BW, Zhang CL and Newman JA: Endotoxin lipopolysaccharide from Escherichia coli and its effects on the phagocytic function of systemic and pulmonary macrophages in turkeys. Avian Dis 35: 901-909, 1991.

6. Esbenshade AM, Newman JH, Lams PM, Jolles H and Brigham KL: Respiratory failure after endotoxin infusion in sheep: Lung mechanics and lung fluid balance. J Appl Physio Respir Environ Exerc Physiol 53: 967-976, 1982.

7. Rojas M, Woods CR, Mora AL, Xu J and Brigham KL: Endotoxin-induced lung injury in mice: Structural, functional and biochemical responses. Am J Physiol Lung Cell Mol Physiol 288: L333-L341, 2005.

8. Matuschak GM and Lechner AJ: Acute lung injury and the acute respiratory distress syndrome: Pathophysiology and treatment. Mo Med 107: 252-258, 2010.

9. Cross CE, Forte T, Stocker R, Louie S, Yamamoto Y, Ames BN and Frei B: Oxidative stress and abnormal cholesterol metabolism in patients with adult respiratory distress syndrome. J Lab Clin Med 115: 396-404, 1990.

10. Sabarirajan J, Vijayaraj P and Nachiappan V: Induction of acute respiratory distress syndrome in rats by lipopolysaccharide and its effect on oxidative stress and antioxidant status in lung. Indian J Biochem Biophys 47: 278-284, 2010.

11. Blackwell TS, Blackwell TR, Holden EP, Christman BW and Christman JW: In vivo antioxidant treatment suppresses nuclear factor-kappa B activation and neutrophilic lung inflammation. J Immunol 157: 1630-1637, 1996.

12. Gordaliza M: Natural products as leads to anticancer drugs. Clin Transl Oncol 9: 767-776: 2007.

13. Sheen WS, Tsai IL, Teng CM and Chen IS: Nor-neolignan and phenyl propanoid from Zanthoxylum ailanthoides. Phytochemistry 36: 213-215, 1994.

14. Yang Z, Hon MH, Chui KY, Xu HM, Lee CM, Cui YX, Wong HNC, Poon CD and Fung BM: Naturally occurring benzofuran: Isolation, structure elucidation and total synthesis of 5-(3-hydroxypropyl)-7-methoxy-2-(3'-methoxy-4'hydroxy phenyl)-3-benzo [b]furancarbaldehyde, a novel adenosine A1 receptor ligand isolated from salvia miltiorrhiza bunge (danshen). Tetrahedron Lett 32: 2061-2064, 1991.
15. Zhou L, Zuo Z and Chow MS: Danshen: An overview of its chemistry, pharmacology, pharmacokinetics, and clinical use. J Clin Pharmacol 45, 1345-1359: 2005.

16. Yuan SL, Wei YQ, Wang XJ, Xiao F, Li SF and Zhang J: Growth inhibition and apoptosis induction of tanshinone II-A on human hepatocellular carcinoma cells. World J Gastroenterol 10: 2024-2028, 2004.

17. Sung HJ, Choi SM, Yoon Y and An KS: Tanshinone IIA, an ingredient of Salvia miltiorrhiza BUNGE, induces apoptosis in human leukemia cell lines through the activation of caspase-3. Exp Mol Med 31: 174-178, 1999.

18. Hua Y, Li W, Wan-Liang S, Lei W, Zai-Liang Y, Yuan L, Ke Z, Ying $\mathrm{W}$ and Wei-Jing $\mathrm{Z}$ : The green tea extract epigallocatechin-3-gallate inhibits irradiation-induced pulmonary fibrosis in adult rats. Int J Mol Med 34: 92-102, 2014.

19. Ford J, Jiang M and Milner J: Cancer-specific functions of SIRT1 enable human epithelial cancer cell growth and survival. Cancer Res 65: 10457-10463, 2005.

20. Dushianthan A, Grocott MP, Postle AD and Cusack R: Acute respiratory distress syndrome and acute lung injury. Postgrad Med J 87: 612-622, 2011.

21. Gattinoni L, Bombino M, Pelosi P, Lissoni A, Pesenti A, Fumagalli $\mathrm{R}$ and Tagliabue M: Lung structure and function in different stages of severe adult respiratory distress syndrome. JAMA 271: 1772-1779, 1994.

22. Mu E, Ding R, An X, Li X, Chen S and Ma X: Heparin attenuates lipopolysaccharide-induced acute lung injury by inhibiting nitric oxide synthase and TGF- $\beta /$ Smad signaling pathway. Thromb Res 129: 479-485, 2012

23. Ni YF, Tian F, Lu ZF, Yang GD, Fu HY, Wang J, Yan XL, Zhao YC, Wang YJ and Jiang T: Protective effect of nicotine on lipopolysaccharide-induced acute lung injury in mice. Respiration 81: 39-46, 2011

24. Ma Z, Ji W, Fu Q and Ma S: Formononetin inhibited the inflammation of LPS-induced acute lung injury in mice associated with induction of PPAR gamma expression. Inflammation 36: 1560-1566, 2013.

25. Torun AN, Kulaksizoglu S, Kulaksizoglu M, Pamuk BO, Isbilen E and Tutuncu NB: Serum total antioxidant status and lipid peroxidation marker malondialdehyde levels in overt and subclinical hypothyroidism. Clin Endocrinol (Oxf) 70: 469-474, 2009.

26. Van Lent PL, van de Loo FA, Holthuysen AE, van den Bersselaar LA and Vermeer H: Major role for interleukin-1 but not tumor necrosis factor in early cartilage damage in immune complex arthritis in mice. J Rheumatol 22: 2250-2058, 1995.

27. Lee SJ and Kim MM: Resveratrol with antioxidant activity inhibits matrix metalloproteinase via modulation of SIRT1 in human fibrosarcoma cells. Life Sci 88: 465-472, 2011.

28. Niederer F, Ospelt C, Brentano F, Hottiger MO, Gay RE, Gay S, Detmar M and Kyburz D: SIRT1 overexpression in the rheumatoid arthritis synovium contributes to proinflammatory cytokine production and apoptosis resistance. Ann Rheum Dis 70: 1866-1873, 2011. 